\title{
La ruptura social como enfermedad: Interpretación mapuche de los conflictos sociales surgidos a partir de un proyecto hidroeléctrico
}

\author{
Cristhian Carrere \\ Master en antropología \\ Consultor Cartis Investigación estratégica \\ e-mail: carrere.cristian@gmail.com
}

\begin{abstract}
Resumen
Este estudio busca describir y analizar una de las aristas de los conflictos sociales surgidos en una comunidad mapuche, al sur de Chile, debido a los impactos de un proyecto hidroeléctrico. La investigación se enfoca en cómo los habitantes locales interpretan y experimentan estos conflictos desde el campo de la salud y la corporeidad.
\end{abstract}

\section{Introducción}

Al sur de Chile, en la comuna de Panguipulli, hay un pequeño lago cordillerano llamado Neltume, ubicado en las cercanías de la frontera con Argentina. Alrededor de éste viven numerosas familias mapuches, constituidas en comunidades y dedicadas principalmente a la pequeña producción agrícola.

Desde el año 2000 aproximadamente, Endesa -principal transnacional energética en Latinoamérica- proyecta construir en el lago una central hidroeléctrica de paso ${ }^{1}$, lo que traería una serie de impactos a nivel local. El proyecto aún no ha sido aprobado, pero la empresa ya se encuentra posicionada en la zona de manera permanente a través de una oficina encargada de establecer relaciones con las comunidades afectadas.

Este estudio es un pequeño análisis crítico del impacto social que ha tenido la presencia de Endesa en la zona. En efecto, si bien el proyecto hidroeléctrico está recién en la etapa de evaluación, éste ya ha originado una serie de perjuicios sociales irreversibles. En el 2003, debido a una serie de conflictos relacionados con la central, la comunidad Juan Quintumán principal comunidad afectada- se dividió en dos: la actual comunidad Juan Quintumán y la nueva comunidad Inalafquén. La principal característica de la división es que ésta refleja una supuesta diferencia de opinión respecto a la construcción del proyecto hidroeléctrico. Diciéndolo de manera

\footnotetext{
${ }^{1}$ Debido a las características de la central, los opositores al proyecto contestan de que se trate de una central de paso, como lo pretende Endesa, y aseguran de que se trata de una central de alta presión.
} 
esquemática, la comunidad Juan Quintumán sería "favorable" a la central, mientras que la comunidad Inalafquén sería "opositora".

Desde la inauguración de la oficina local de Endesa, las comunidades del lago Neltume - incluidas las dos mencionadas anteriormente - se han convertido en un verdadero campo de batalla entre estas dos posturas contrarias. Una lucha que ha desembocado en una ruptura social. Un quiebre de los vínculos familiares y vecinales; y del cual atestan numerosos casos de discriminación y de exclusión, cometidos y padecidos a la vez, por los mismos habitantes locales.

En el siguiente artículo exploraremos la interpretación local que se tiene de esta ruptura social. Es a la vez un estudio sobre el discurso y sobre la experiencia corporal de los conflictos sociales que llevaron a la división de la comunidad Juan Quintumán. En efecto, la ruptura de las relaciones sociales pareciera experimentarse en la corporeidad misma de las personas, puesto que dicha ruptura es asociada permanentemente a algún tipo de patología física, la que podría llevar eventualmente a la muerte. El deterioro del cuerpo o el fin de la vida, son concebidos como consecuencias de un deterioro en las relaciones sociales; un verdadero "envenenamiento" de los lazos en la comunidad, el cual traería efectos negativos en la salud de sus integrantes.

\section{Metodología}

Este artículo es el resultado de un estudio de investigación independiente que realizamos en la localidad de Neltume y en la rivera del lago Neltume los meses de noviembre y diciembre del 2012. Posteriormente a esos 2 meses de trabajo de campo, volvimos en varias ocasiones por cortas estadías durante el invierno del 2013.

La metodología de investigación fue exclusivamente un trabajo de inmersión por observación directa y observación participante.

Durante el primer mes de trabajo de campo se realizaron varias estadías, cada una de varios días. Se establecieron las primeras relaciones y participamos en numerosas discusiones sobre la problemática de la central. Los intercambios dieron lugar a relaciones más duraderas y se establecieron vínculos con dos familias en particular: la familia de don Raúl Huenún y la familia de don Roberto Catrimán².

\footnotetext{
${ }^{2}$ Los nombres y apellidos han sido modificados, pues no obtuvimos la autorización de las personas implicadas antes de la publicación de este artículo.
} 
Durante el segundo mes de trabajo de campo, las estadías en Neltume se hicieron más largas. Las conversaciones sobre la central fueron más seguidas y se entró en contacto con personas más activas en la oposición al proyecto hidroeléctrico. Hoy en día resulta imposible -y sin duda innecesario para los fines de este artículo- enumerar los encuentros, conversaciones, intercambios de información, etc., que tuvimos en Neltume, los cuales han ido nutriendo los análisis que aquí exponemos.

Finalmente, cabe resaltar que durante el trabajo de campo ninguna conversación fue grabada. Las citas aquí reproducidas son una transcripción posterior realizada de memoria. Por ende, éstas podrían tener ciertas diferencias con respecto al diálogo original. Sin embargo, nos preocupamos, evidentemente, de guardar el sentido y describir el contexto.

\section{El contexto histórico y el proyecto hidroeléctrico}

Antes de dar inicio al desarrollo de la problemática que nos atañe, expondremos brevemente el contexto histórico local y explicaremos a grandes rasgos en qué consiste el proyecto hidroeléctrico, así como su impacto ambiental más importante. Este capítulo nos permitirá situar adecuadamente nuestras reflexiones, las cuales no pretendemos generalizables al mundo mapuche, ni ambicionamos extrapolar hacia otros casos de impacto socioambiental.

\section{La historia de Neltume}

Neltume es una zona que ha tenido una historia muy convulsionada, marcada de conflictos, violencia y una profunda crisis laboral a partir de los años $1970^{3}$.

Hasta comienzos del siglo $\mathrm{XX}$, gran parte de la región era territorio mapuche. Panguipulli era, en efecto, una de las comunas de mayor densidad indígena del país (Skewes et al, 2012). La gran mayoría de las familias se habían ido refugiando en estas tierras cordilleranas desde la colonia, huyendo del avance progresivo de la propiedad agrícola que se iba apoderando de los llanos del valle central, donde se encuentran las mejores tierras agrícolas.

El avance del latifundio no se detuvo por ello en las puertas de Los Andes, sino que siguió avanzando progresivamente, absorbiendo en cada uno de

\footnotetext{
${ }^{3}$ Una buena parte de la violenta historia que ha remecido a Neltume no será citada en este artículo, pues no es atingente a la problemática que nos atañe. Sin embargo, recomendamos consultar los trabajos que se han publicado al respecto, como, por ejemplo: Comité Memoria de Neltume (2003), CODEPU (1992), Toledo (1994), Millanguir (2007), Skewes, Solari, Guerra, \& Jalabert (2012).
} 
sus pasos tierras originalmente mapuches. Fue así como a principios del siglo XX la comuna de Panguipulli experimentó un auge económico de relevancia (Bernedo, 1994). Una importante colonia europea ${ }^{4}$ se instaló y el comercio y la producción agrícola y maderera creció considerablemente. Grandes extensiones de tierra en Neltume fueron compradas por colonos, quienes se dedicaron a explotar el bosque durante décadas. Los colonos alemanes, franceses y chilenos tomaron posesión de las tierras mapuches a través de transacciones financieras que ignoraban la existencia de habitantes locales o simplemente a través de robos y asesinatos. Tal fue el caso de Joaquín Mera, un personaje que sembró el terror entre la población local (Diaz, 2005 [1907]).

A principios del siglo $\mathrm{XX}$, los mapuches habían sido expulsados de sus tierras y acantonados en pequeñas comunidades, entre ellas la comunidad Juan Quintumán ${ }^{5}$. En Neltume, el tamaño de las propiedades agrícolas mapuches, pequeñas y empinadas, condicionaron su deterioro: se erosionaron y empobrecieron rápidamente por la falta de rotación y la sobreexplotación. Impedidos de solventar sus necesidades básicas a través de la producción agrícola independiente, los mapuches se convirtieron en empleados de los grandes fundos madereros.

Finalmente, a comienzos de la década de 1970 la madera se acabó. El bosque fue sobreexplotado y vino así una profunda crisis laboral que llevó a mucha gente a emigrar. Neltume se hundió desde entonces en una profunda crisis económica de la cual no volvería a levantarse.

\section{La central de Endesa}

La intención de crear una central hidroeléctrica en Neltume data exactamente de la misma fecha que la crisis económica, sin embargo, no fue hasta el 2000 que ésta se concretó. El proyecto de Endesa es relativamente pequeño si tomamos en cuenta el voltaje que se pretende producir 6 . Sin embargo, el trabajo que éste implicaría es considerable. Si el proyecto se aprueba, se instalaría una bocatoma en el nacimiento del río Fuy, en el lago Pirihueico. De ahí, el agua se encaminaría a través de una tubería subterránea que atravesaría el gran fundo Neltume. La tubería

\footnotetext{
${ }^{4}$ La gran mayoría de los autores (por ejemplo: Bernedo (1994), Millanguir (2007), Skewes, Solari, Guerra, \& Jalabert (2012), etc.) considera principalmente la colonización europea, debido a que fue la más importante en cifras y en aporte económico (la mayoría de las familias burguesas eran de origen alemán y vasco francés). Sin embargo, también hubo una importante colonia de inmigrantes de otras partes del mundo, como sirios y otomanes.

${ }^{5}$ En la comuna de Panguipulli la llamada "radicación" comenzó en 1908 y terminó 2 años después. Se habrían entregado 202 títulos de merced, equivalentes a 37,7 mil hectáreas aproximadamente, para un total de 3,188 personas (De La Maza, 2005, p. 109).

6 Potencia aproximada de 490 MW y una generación media anual estimada en 1.885 GWh. $\underline{\text { http://seia.sea.gob.cl/expediente/ficha/fichaPrincipal.php?modo=ficha\&id_expediente }=5124693}$
} 
llegaría de esta manera a la parte alta de la comunidad Juan Quintumán, y de allí bajaría en caída libre hasta las turbinas que producen la electricidad. Todo ello bajo tierra. Finalmente, las aguas se expulsarían en el lago Neltume, provocando un aumento de la cota de hasta 1 metro en época de lluvias ${ }^{7}$.

Según Elías Sandoval ${ }^{8}$, encargado de la RSE de Endesa en Neltume, la comunidad Juan Quintumán sería la más afectada de todas, pues allí se encontrarían las tres obras descubiertas de la central ${ }^{9}$. Seis son las familias de esta comunidad impactadas directamente. Parte de sus propiedades agrícolas serían en efecto permutadas por tierras que Endesa adquirió en un sector llamado Remeco, en la parte alta de Juan Quintumán ${ }^{10}$.

Sin embargo, el mayor impacto producido por la central, y el que provoca mayor temor, no consiste en la expropiación, sino en el aumento del nivel del lago Neltume debido a las aguas del río Fuy vertidas por la central. Según los habitantes de la rivera del lago, el aumento del nivel del lago podría conllevar graves problemas, pues sufren ya de grandes inundaciones en invierno ${ }^{11}$.

\section{El impacto social y sus vivencias}

Ahora que expusimos a grandes rasgos el crítico contexto histórico y explicamos brevemente en qué consiste la central y su principal impacto ambiental - el aumento del nivel del lago -, podemos volcarnos hacia la problemática que nos interesa específicamente: la ruptura social provocada por el proyecto de Endesa y la interpretación y experiencia de los conflictos sociales como patologías físicas.

\section{La división de la comunidad Juan Quintumán}

\footnotetext{
7 Sin embargo, si luego de llegar a 1 metro el lago sigue subiendo debido a las lluvias, Endesa se desresponsabilizaría totalmente. Según las explicaciones de Elías Sandoval, encargado de la RSE de Endesa en Neltume, la empresa no asumiría las consecuencias del aumento del lago, pues éste "no [habría] subido por las aguas vertidas directamente por la central" (Elías Sandoval). Una actitud que, dicho sea de paso, podríamos calificar a lo menos de irresponsable y que algunos habitantes locales consideran simplemente mal intencionada.

${ }^{8}$ Entrevistas realizadas en la oficina de Endesa en Netume.

${ }^{9}$ Una chimenea de equilibrio, el patio de mufas y el camino de acceso al patio de mufas, a los túneles y a la caverna de máquinas.

${ }^{10}$ Vale la pena recordar que, debido al impacto económico del turismo en el precio de la propiedad, las tierras cercanas a los lagos tienen un valor mayor a las tierras agrícolas y muchas veces mayor a las tierras agrestes, sin potencial agrícola, como lo son las tierras de Remeco. Podemos preguntarnos por ende, ¿cómo es posible que aquellas tierras adquiridas por Endesa, hayan sido evaluadas como "equivalentes" a las actuales propiedades mapuches?, sabiendo que la ley Indígena 19.253, artículo 13, obliga a las empresas a entregar tierras de igual valor a las tierras expropiadas.

${ }^{11}$ Una de estas consecuencias es la desaparición del humedal del río Cuacúa, el cual desemboca en la rivera norte del lago, y que es el hábitat de numerosas especies de pájaros y de flora endémica.
} 
Como en todos los casos donde se pretende realizar grandes proyectos de desarrollo, las relaciones sociales entre habitantes locales se han tensionado debido al proyecto hidroeléctrico de Neltume; nuevos conflictos han surgido entre vecinos y aquellos que precedían al proyecto tendieron a agravarse.

En esta situación que podríamos catalogar momentáneamente como saturada de estrés social (Sandín, 1999), las opiniones y posiciones con respecto al proyecto que afecta a la comunidad han tendido a "solidificarse". Por un lado, oponerse al proyecto pareciera implicar el fin de las relaciones con la empresa, sus representantes e incluso con toda persona que a su vez tenga algún vínculo con Endesa; so pena de ser etiquetado de "endesino", "vendido" o peor incluso, de "soplón" por el resto de los opositores. Por otro lado, ser favorable al proyecto tiene igualmente efectos adversos. Al igual que en el caso anterior, el posicionamiento pareciera haberse "solidificado". Ser favorable al proyecto implicaría cortar toda relación con personas opositoras; so pena, una vez más, de ser etiquetado y discriminado esta vez por el resto de los habitantes favorables al proyecto.

Ha surgido así, una situación que podríamos catalogar de "radical", en el sentido en que sólo aparecen como socialmente posibles dos posiciones irreconciliables y donde cada quien pareciera cortar relaciones con personas que hoy declaran tener opiniones contrarias ${ }^{12}$.

Según las mismas personas con quienes pudimos conversar, sería esta misma situación -de "solidificación" de las opiniones y posiciones con respecto al proyecto de Endesa- la que habría llevado a la comunidad Juan Quintumán a la cisión ${ }^{13}$. Un claro ejemplo de ello es el caso de Roberto Catrimán, el lonko de la comunidad Juan Quintumán. En un comienzo, Roberto Catrimán era contrario a la realización del proyecto hidroeléctrico. Sin embargo, con el paso del tiempo su postura fue cediendo a favor de la empresa y terminó por abandonar los rangos de la oposición. La decisión de Roberto Catrimán implicó que éste aceptara relacionarse con los

\footnotetext{
${ }^{12}$ Podría resultar necesario recordar que esta hipótesis de "solidificación de las posturas y opiniones" hace referencia a una situación social, y en ningún caso es una reflexión sobre la capacidad de análisis de las personas de manera individual. Si bien creemos que existe una situación de "solidificación de opiniones", las personas pueden evidentemente tener reflexiones independientes y libres de toda presión respecto a la central. A nuestro parecer, un ejemplo de ello es el presidente de la comunidad Valeriano Cayicul, comunidad colindante de Juan Quintumán. Éste se opone al proyecto pero asiste a las reuniones informativas que da Endesa, se entrevista tanto con los responsables del proyecto como con la gente que milita contra éste. Dice no discriminar a nadie según la opinión que tenga respecto a la central y se opone a su construcción pues asegura, a partir de su propia experiencia en el rubro de la construcción de grandes proyectos de desarrollo, que la información que entrega Endesa es tergiversada. Su comportamiento poco habitual, le ha valido sin embargo, críticas y sospechas por parte de algunos opositores al proyecto.

${ }^{13}$ Sería interesante -y tal vez incluso necesario- indagar en otra ocasión sobre las razones que llevaron a esta "solidificación" de las posiciones, y dar cuenta del rol y responsabilidad de Endesa y su política de RSE en la situación actual.
} 
representantes de Endesa en Neltume y, sobre todo, que aceptara recibir las donaciones y servicios ofrecidos por la empresa en el marco de su RSE, lo que le costó severas críticas.

Roberto Catrimán justificó su cambio de posición como el resultado inevitable de un combate que consideraba como perdido de antemano, debido a las fuertes desigualdades de capitales (económico y social) que separan a la empresa de los habitantes locales. "Al final de cuentas, igual van a terminar haciendo la central" -nos decía don Roberto con tono de derrota.

El cambio de postura del representante tradicional de la comunidad Juan Quintumán vino a dar el golpe de gracia a una situación ya precaria entre las personas favorables y los opositores al proyecto, lo que condujo finalmente a la división definitiva y oficial de la comunidad. La parte sur de la antigua comunidad mantuvo el nombre Juan Quintumán, sus representantes -organizados en las denominadas Mesas de Trabajoaccedieron a firmar un acuerdo de colaboración con la empresa y quedó, en la opinión pública, catalogada desde entonces como una comunidad "proEndesa" o "endesina"14. Del otro lado, en la parte norte, se creó la nueva comunidad Inalafquén, sus representantes rechazaron las conversaciones con la empresa y se constituyó así, desde su fundación, como una comunidad "opositora" al proyecto ${ }^{15}$.

\section{Dos familias enfrentadas a la enfermedad}

Esta ruptura social provocada por el proyecto hidroeléctrico de Endesa se expresa en la vida social a través de múltiples dimensiones. Nosotros, tal como lo anunciamos en la introducción, nos focalizaremos en una sola dimensión: la manera en que la enfermedad y la muerte, en definitiva el deterioro del cuerpo individual, es interpretado como el resultado de una situación social conflictiva, es decir el deterioro de lo que vendría a ser el cuerpo social.

Desarrollaremos esta reflexión a través de dos ejemplos: las dificultades de salud enfrentadas por la familia de don Raúl Huenún y aquellas enfrentadas por la familia del lonko Roberto Catrimán. Veremos que ambos casos muestran grandes semejanzas, a pesar de encontrarse en situaciones absolutamente contrarias respecto al proyecto hidroeléctrico.

\footnotetext{
${ }^{14}$ Los detalles de dicho acuerdo se encuentran en: https://www.e-seia.cl/archivos/c15_Anexo_Z.pdf

${ }^{15}$ Tal vez resulte necesario aclarar que hablamos en términos de etiquetaje porque consideramos imposible que una división geográfica calce efectivamente con las diferencias de opinión respecto a la central. Los títulos de "opositora" y "favorable" serían una especia de "presentación oficial" de cada comunidad, forjada a partir de las opiniones de sus respectivos líderes.
} 


\section{La familia Huenún}

El 3 de diciembre del 2012, muchos fuimos a visitar a la familia Huenún. Irma estaba devastada. Lloraba desconsoladamente. Cuando llegamos, se lanzó en los brazos de un pariente para abrazarlo con la determinación de una persona que sufre sin vergüenza. Su padre había muerto hace 2 días, a las seis de la mañana en el hospital de Panguipulli. Sufría un cáncer que se había expandido por todo su cuerpo. Tenía metástasis en las piernas, brazos, cuello, vientre... La señora Norma, la esposa del difunto, estaba más tranquila. Tenía los ojos rojos y serios, pero no lloraba abiertamente como sus hijos. Nos saludó, agradeció nuestra llegada y nos invitó a ingresar a la casa. En el salón, unas diez personas estaban sentadas, pegadas a los muros de la casa y rodeando a don Raúl, quien yacía sin vida detrás del cristal del ataúd. Dimos la vuelta al salón saludando a cada una de las personas, intercambiando una pequeña sonrisa cordial y triste. Luego nos sentamos en las sillas que nos trajeron y nos quedamos callados. En la cocina había mucho movimiento, Irma había tenido que dejar de llorar para ocuparse de las visitas.

Habíamos conocido a la familia Huenún hace ya un par de meses. Estábamos al tanto de la enfermedad de don Raúl, y Pedro Cardyn, médico, amigo y compañero de don Raúl en la lucha contra la central, nos había confesado que su estado de salud era grave e irreversible.

Aquí estamos enfermos en esta casa -nos decía doña Norma, haciendo referencia a la enfermedad de su marido-. ¿Cómo podemos luchar contra la central? Si eso es lo que lo tiene enfermo: la central. Todas estas peleas. El 'estrés'. Porque él se oponía. Siempre estaba ahí en las reuniones. Iba para todos lados. Al final estaba cansado. Se fue cansando, se fue enfermando. Es por todo esto que está así, en el hospital.

Antes que don Raúl Huenún falleciera, doña Norma casi no hablaba de otra cosa. Todo giraba en torno al proyecto de la central, los conflictos sociales que había causado, el "estrés" y -según ella- sus efectos más inmediatos y determinantes: la enfermedad y una muerte que ya parecía tocar a la puerta.

\section{La familia Catrimán}

Para la familia Catrimán, en el otro extremo de este restringido mapa de opiniones sobre la central, la situación es muy semejante a la experimentada por la familia Huenún.

Con Pedro Catrimán, hijo del lonko Roberto Catrimán, las conversaciones siempre fueron íntimas desde el comienzo. Como alguien que no pierde el 
tiempo en futilidades protocolares antes de lanzar sus más terribles dolores a quemarropa.

- La vida está llena de sorpresas -dice Pedro en voz alta pero como hacia adentro-. A mí me mataron a mi niña... por tanto conflicto, pues oiga. Mi hija. Era una niña no más.... Tres años tenía.

- ¿Cómo ocurrió? - pregunto lento.

- Así no más poh'. La mataron. -y luego de un silencio sin molestia y sopesando la confianza que me entregaba-:

- ¿Pasó hace tiempo?

- ¡Sí, hace un tiempo ya.

- ...Una niña.

- Claro... Chiquita mi hija. Yo la quería tanto y esos me la mataron.

- ¿Quiénes esos?

- Los de allá -dice alzando el mentón hacia el norte.

- ¿Quién vive allá?

- La gente de Inalafquén.

- iCCómo?! - pregunto asombrado.

- Así no más. Cayó pues oiga

- ¿Cómo eso que cayó?

- No sé, como un animal [...] como un toro. La mataron así no más [...] murió al tiro. Si era niña, no tenía fuerza.

Según el relato de Pedro, su hija habría fallecido alcanzada por un "mal de ojo" que éste atribuye a la gente de Inalafquén. Un drama ocasionado, según las mismas explicaciones de Pedro, por los conflictos sociales, los mismos que desembocaron en la división de la antigua comunidad Juan Quintumán.

Lamentablemente, no sólo la muerte de la hija de Pedro ha marcado a la familia Catrimán: el mismo lonko sufre de una enfermedad que lo ha ido desgastando poco a poco. Ha tenido que hospitalizarse en varias ocasiones y sufre de unos quistes que no le permiten moverse con facilidad. Un par de semanas después de que don Roberto nos confiara sus males, su esposa nos confió que su salud se había deteriorado aún más; y peor aún: Inés, su nuera - quien vive en la casa contigua - estaba en cama, devastada, pues acababa de perder a un niño que no alcanzó a dar a luz. Todo "debido a la central [...] Todo por culpa del 'estrés', de las peleas", dice la mujer de don Roberto con voz de sentencia. 
La familia Catrimán pareciera hundirse en la enfermedad y en la muerte. Y esta situación es atribuida sistemáticamente a los conflictos que tienen contra el resto de los habitantes del lago: aquellos que se oponen al proyecto hidroeléctrico. Al igual que para doña Norma -la esposa del difunto don Raúl Huenún-, para la familia Catrimán la enfermedad y la muerte parecieran ser directamente consecuencias de la disolución social. Culpa de la ruptura y del envenenamiento de las relaciones. Como si el quiebre de los lazos interpersonales pusiera en jaque el precario equilibrio en el que yace nuestro cuerpo. Como si el disfuncionamiento del cuerpo físico fuese el reflejo de lo que es considerado el disfuncionamiento del cuerpo social.

\section{El lago, la salud y la feminidad}

Los casos de la familia Huenún y Catrimán muestran de manera bastante clara el vínculo que se establece entre la salud/enfermedad y las relaciones sociales. En el caso de la familia Huenún, la enfermedad es atribuida de manera muy general a la situación precaria de la convivencia en el lago. En el caso de la familia Catrimán, es derechamente interpretada como un acto de violencia, directo y voluntario. Pensar que la enfermedad que golpea a uno de nuestros seres queridos es culpa del "estrés" -provocado por una situación social precaria- es evidentemente diferente a creerla como el resultado de un acto maligno, voluntario y mágico -como es el caso del "mal de ojo". Analizar el carácter sobrenatural o mágico de este último caso supondría un tiempo con el que aquí no contamos. Por el momento, nos contentaremos con dar cuenta de aquello que ambos discursos comparten: en ambos casos la enfermedad y la muerte son interpretadas como el resultado de una situación social patológica, cuya principal característica sería un deterioro en las relaciones interpersonales.

\section{La noción clave de "equilibrio"}

Esta relación entre salud y convivencia está dada principalmente por un concepto clave que une, en el pensamiento mapuche, tanto la dimensión social como la individual: el concepto de equilibrio. (Grebe, Pacheco, \& Segura, 1972). Según Ana Mariella Bacigalupo (1998) - investigadora que citaremos ampliamente más adelante - el desequilibrio social correspondería a una situación de ruptura social, mientras que el equilibrio sería atribuido a un estado de unión social ${ }^{6}$. En la dimensión individual, el

\footnotetext{
${ }^{16}$ Si bien Ana Mariella Bacigalupo no desarrolla estas nociones, sí se apoya sobre ellas para desarrollar su propia problemática. Por otro lado, vale la pena mencionar que nosotros consideramos el concepto de unión socialcomo una categoría ideal, la cual representaría un estado sociológico inexistente pero deseable; pues, como ya bien lo ha desarrollado la antropología dinámica, no existen sociedades sin conflictos ni sociedades sin desigualdades (Balandier, 2010 [1976]).
} 
desequilibrio correspondería a la enfermedad y el equilibrio a la salud. De esta manera, sintetizando, el desequilibrio provocado en las relaciones sociales por culpa del proyecto hidroeléctrico, sería, bajo esta perspectiva, la principal causa de enfermedad; la enfermedad siendo considerada, a su vez, como un desequilibrio individual, es decir como un desequilibrio interno de la persona.

Una serie de investigaciones muestran esta relación entre la salud/enfermedad y las condiciones de convivencia social a través de la noción de equilibrio. Un estudio de Hassen \& Cortez (2012) realizado precisamente en el lago Neltume -pero sobre la salud mental de sus habitantes- parte desde una línea interesante, basándose en la noción mapuche de küme mogñen o "buen vivir". El küme mogñen es el resultado de un equilibrio entre tres realidades: el individuo, la comunidad y la naturaleza. Tres elementos interconectados que forman una triangulación y de la cual dependería tanto la salud de las personas como el futuro de la comunidad. De manera general, en la cosmovisión mapuche prima de manera trascendental esta noción de equilibrio (Grebe, 1971), verdadera condición sine qua non para la existencia del mundo. Así, desde esta perspectiva, cuando la comunidad se ha visto afectada - "cuando el ambiente dentro de la familia es de discordia, hay peleas entre vecinos, etc." (Hassen \& Cortez, 2012, p. 583) - o cuando un acontecimiento remueve a la naturaleza -por la intervención humana directa, como la construcción de una central, o por un acontecimiento natural, como una inundación- se provoca un desequilibrio en el mundo, lo que tiene un impacto en la triangulación y por ende en el individuo, afectando eventualmente su propio equilibrio individual, es decir su salud física.

\section{El rol de la naturaleza}

Vimos en los dos ejemplos citados anteriormente cómo los actores relacionan efectivamente la salud y la enfermedad con las condiciones de convivencia, ello a través de un criterio de equilibrio/desequilibrio. Sin embargo, aún falta dar cuenta de cómo, en el pensamiento mapuche, la naturaleza influye en la salud de las personas. Es decir: ¿qué rol ocupa la naturaleza en la triangulación? Los autores Hassen \& Cortez no desarrollan en su artículo ninguna reflexión al respecto, limitándose a asegurar -sin explicar- que los impactos en la naturaleza tienen un impacto en la salud de los individuos. Muchos otros estudios sin embargo, sí han analizado pero en otros terrenos- el rol de la naturaleza en esta relación de interdependencia armoniosa entre el individuo, su comunidad y su entorno natural. Nosotros no pretenderemos desarrollar todas las dimensiones de 
esta triangulación, pero sí esperamos aportar un elemento más en la reflexión sobre el caso de Neltume.

Ana Mariella Bacigalupo (1998), en sus investigaciones sobre las machis ${ }^{17}$ a través de una perspectiva de género, nos entrega las bases para reinterpretar el rol de la naturaleza en la situación social actual de las comunidades del lago Neltume. En primer lugar, debemos mencionar que en la cosmogonía mapuche, a los elementos de la naturaleza se les adjudica un sexo y se les atribuyen todas o algunas de las características de dicho género. El sol, el rayo y los volcanes, por ejemplo, son elementos masculinos y se les asocia con el "despertar repentino de energía" (Bacigalupo 1998), es decir, con el vigor. Por otro lado, la luna, las estrellas y los lagos son femeninos, y se los relaciona con los vínculos sociales: las relaciones familiares y los lazos entre miembros de una misma comunidad. Vemos así un primer vínculo entre la naturaleza, el individuo y su comunidad: dotados de características sexuadas, los elementos de la naturaleza -sol, lago, luna, etc.,- comparten, en la cosmogonía mapuche, características comunes a los humanos. Así, según Bacigalupo, los lagos están relacionados con la feminidad, la cual a su vez está relacionada con los "vínculos sociales"; o más aún, con la "unificación" de la sociedad mapuche. Este último concepto toma para nosotros una importancia mayor. Recordemos que en Neltume no sólo existen peleas entre "opositores" y "favorables" al proyecto, sino que los conflictos desembocaron en una división administrativa de la comunidad.

El análisis de la percepción mapuche del mundo, a través de un análisis de género, nos revela así una relación simbólica directa entre el lago, la comunidad y la salud: las enfermedades o desequilibrios individuales serían así considerados como efectos de un desequilibrio social, y éste último, a su vez, como efecto de un proyecto hidroeléctrico que pretende provocar un desequilibrio natural en el lago, un elemento asociado justamente a la noción de unión social.

Resumiendo, en Neltume las tres partes de la triangulación se encontrarían en algún estado de desequilibrio: los individuos enfermos, la comunidad dividida en dos y la naturaleza en un estado que podríamos llamar de "desequilibrio potencial" o, lo que vendría a ser lo mismo, bajo un "riesgo de desequilibrio"18.

\section{El Nguillatún}

\footnotetext{
${ }^{17}$ Chamán mapuche.

${ }^{18}$ Resulta necesario recordar que el lago aún no ha sido intervenido, y que lo que existe por ende es un "riesgo de desequilibrio".
} 
La "coincidencia" de que las tres partes de la triangulación del cosmos mapuche se encuentren en un estado de desequilibrio -el individuo, la sociedad y la naturaleza-, muestra una resonancia entre estas tres dimensiones, una interconexión o, si se prefiere, una conectividad simbólica. Sin embargo, ¿cómo las personas y la comunidad se relacionan simbólicamente con el lago y los otros elementos de la naturaleza?, ¿cómo es posible, en la reflexión mapuche, esta resonancia?

La respuesta está en el nguillatún ${ }^{19}$. Entregando una definición breve, diríamos que el nguillatún es el principal rito mapuche y que corresponde a una rogativa donde se les pide a los seres superiores el bienestar, la unión de la comunidad y la fertilidad de la tierra (Grebe, 1971). El nguillatún es el rito a través del cual el hombre actualiza su relación con el mundo espiritual, asegurando la continuidad del tan preciado equilibrio del cosmos. En definitiva, el nguillatún es precisamente el rito que permite la resonancia simbólica entre las personas, su entorno social y su entorno natural.

Ahora bien, el nguillatún no se realiza en cualquier lugar, sino en el nguillatuwe, espacio sagrado por excelencia, protector de enfermedades, de catástrofes y de todo mal (Bacigalupo, 1998). Al ser el espacio donde se realiza la rogativa mapuche, el nguillatuwe vendría a ser la expresión territorial de este vínculo, de esta triangulación. Es la expresión espacial de la unión social, de la salud individual y del buen acontecer de los ciclos de la naturaleza.

Como lo mencionamos anteriormente, si la central hidroeléctrica se llegase a construir, el lago Neltume subiría aproximadamente $1 \mathrm{mt}$ de altura, lo que implicaría la inundación del nguillatuwe de las comunidades del lago, el cual se encuentra en una playa de la rivera sur del lago. Podemos suponer -tomando en consideración las vinculaciones simbólicas que hemos analizado- la percepción de riesgo que los habitantes del lago tienen sobre la posible inundación de este importante espacio de rogativa: hacer desaparecer el nguillatuwe sería hacer desaparecer el espacio donde se realiza el acto que asegura el equilibrio del cosmos, que provee fertilidad a la tierra y unión en la comunidad.

A pesar de encontrarse recién en la etapa de evaluación, el proyecto hidroeléctrico de Neltume pareciera impactar en definitiva, todas las dimensiones aquí analizadas:

\footnotetext{
${ }^{19}$ Los vínculos entre los habitantes del lago Neltume y el medioambiente son ciertamente numerosos y complejos. En este artículo nos limitaremos a mencionar el nguillatún, principalmente debido a su importancia cultural y al rol estratégico que ha tenido en los conflictos por la central.
} 
- En primer lugar, analizamos el impacto directo, ocasionado a nivel social y que podríamos considerar como un impacto fácilmente objetivable: el envenenamiento de las relaciones sociales a partir de la "solidificación" de las opiniones y la ruptura de la comunidad Juan Quintumán.

- En segundo lugar, vimos cómo se desprende, a partir de aquí, un impacto a nivel individual, correspondiente a la salud física y psíquica de los miembros de la comunidad, y que nosotros interpretamos como una representación y experiencia específica del impacto social, es decir, del impacto objetivable.

- En tercer lugar, abordamos el riesgo de impacto ambiental del proyecto, y dimos cuenta de qué manera el aumento del nivel del lago -el lago como actor provisto de características sociales femeninastermina cerrando el ciclo de desequilibrios provocados en la triangulación individuo-sociedad-naturaleza, tan presente en el pensamiento mapuche.

- Finalmente, el análisis de los vínculos simbólicos existentes entre la sociedad, el individuo y la naturaleza nos permitió entender el alcance que tiene efectivamente la amenaza de desaparición del espacio ritual que permite, en la cosmogonía mapuche, que estas tres dimensiones puedan relacionarse de manera armoniosa.

\section{Conclusiones}

Durante nuestra reflexión, tratamos de desembrollar algunos de los numerosos hilos con que se construyen los discursos en el lago Neltume respecto al proyecto hidroeléctrico de Endesa. Tratamos de entender algunos de los lazos que unen, en la reflexión mapuche, los conceptos de enfermedad, equilibrio, unión social e impacto ambiental. Para ello tomamos un pequeño desvío a través de la perspectiva de género, la que nos permitió darle una nueva lectura al impacto que tendría la central en el lago y la eventual inundación del espacio de rogativa mapuche, nguillatuwe.

Sin embargo, debemos advertir que el alcance de nuestra reflexión es limitada, pues la complejidad social en el lago y la complejidad de los conflictos surgidos a partir de la central es considerable. Basta con anunciar que en Neltume existe una multiplicidad de discursos. Por ejemplo, si bien el lago es considerado en la cosmogonía mapuche como un elemento femenino, responsable del mantenimiento de las buenas relaciones sociales, esta caracterización debe ser distinta, por ejemplo, para las personas que asisten regularmente a alguna iglesia pentecostés, numerosas en el lago, o para personas con otras trayectorias de vida 0 
referencias culturales. Igualmente, por dar un segundo ejemplo, la importancia del nguillatuwe puede ser relativizada por algunos actores locales favorables al proyecto, quienes ven como factible su desplazamiento hacia otro sitio ${ }^{20}$. Así, podemos constatar que la realidad social es bastante más compleja que la que aquí describimos y que este artículo tan sólo esboza alguna de sus características, sin pretender dar un retrato acabado de la situación local.

A pesar de esta multiplicidad de discursos y de significaciones, podemos sostener que la coyuntura social en Neltume presenta dos características manifiestas: en primer lugar, la situación conflictiva que buscamos analizar en parte, y que ha llevado a la cisión administrativa de una comunidad. En segundo lugar, la interpretación local del quiebre social como patologías y la vivencia de los conflictos en la corporeidad misma de las personas.

Si bien hablar de "somatización de los conflictos" puede parecer osado, sí creemos pertinente hablar, en este caso, de alguna forma de incorporación de los males sociales. Esta incorporación es tal vez la expresión más íntima y acabada de la presencia excesivamente importante de Endesa en las dinámicas sociales. En efecto, todo en la vida del lago Neltume pareciera girar en torno a la central, a la información y a la desinformación ${ }^{21}$ que circula respecto a su construcción. El proyecto ha venido a ocupar así, todos los rincones de la vida cotidiana; haciéndose omnipresente incluso. Vivido hasta en el rincón más íntimo, hasta en la aflicción de un cuerpo doliente. Una especie de obsesión hidroeléctrica. Fruto de una intervención local que ha convertido a la central en neblina: ubicua y sin embargo empecinada en mantenerse inasible.

\section{Bibliografia}

BACIGALUPO, A. M. (1998). Les chamanes mapuche et l'expérience religieuse masculine et féminine.

BALANDIER, G. (2010 [1976]). Anthropologie Politique. Paris: PUF.

BECKER, H. (1963). Outsiders: Studies In The Sociology Of Deviance. New York: The Free Press.

BERNEDO, P. (1994). Panguipulli: historia a cuatro tiempos. Santiago: Optima.

\footnotetext{
${ }^{20}$ De hecho, el desplazamiento del nguillatuwe sería la intención inicial de Endesa.

${ }^{21}$ No sería exagerado afirmar que los actores divulgan y se guardan la información sobre la central de una manera estratégica.
} 
C. M. (2003). Guerrilla en Neltume. Santiago: LOM.

CODEPU. (1992). Chile. Recuerdos de la Guerra. Valdivia, Neltume, Chihui, Liquiñe. (Vol. 2). Santiago: CODEPU/EMISION.

DE LA MAZA, F. (2005). Política social para indígenas. Un análisis desde la perspectiva del Estado y le interacción local. Tesis para optar al grado de doctor en antropología. México: CIESAS.

DIAZ, A. (2005 [1907]). Parlamento de Coz Coz. Santiago: Imprenta Araucaria.

GREBE, M. E., FERNÁNDEZ, J., \& FIEDLER, C. (1971). Mitos, creencias y concepto de enfermedad en la cultura mapuche. Acta de psiquiatría y psicología para América Latina, XVII(3), 180-193.

GREBE, M. E., PACHECO, S., \& SEGURA, J. (1972). Cosmovisión Mapuche. Cuadernos de la realidad nacional (14), 46-73.

HASSEN, F., \& Cortez, M. (2012). Aproximaciones a la noción mapuche de küme mogñen: equilibrio necesario entre el individuo, su comunidad y la naturaleza. Revista Electrónica de Psicología Iztacala, 15(2), 575-593.

MILLANGUIR, D. (2007). Panguipulli, Historia y Territorio 1850-1946. Valdivia: Imprenta Austral.

SANDÍN, B. (1999). El estrés psicosocial. Madrid: Klinik.

(2003). El estrés: un análisis basado en el papel de los factores sociales. International Journal of Clinical and Health Psychology, 3(1), 141157.

SKEWES, J. C., Solari, M. E., Guerra, D., \& Jalabert, D. (2012). Los paisajes del agua: naturaleza e identidad en la cuenca del río Valdivia. Chungara, Revista de Antropología Chilena, 44(2), 299-312.

TOLEDO, G. (1994). La muerte y sus interpretaciones. Represión política en el complejo forestal y maderero Panguipulli LTDA. 1973. Informe de Práctica Profesional para optar al título de Antropólogo. Valdivia: Universidad Austral de Chile. 